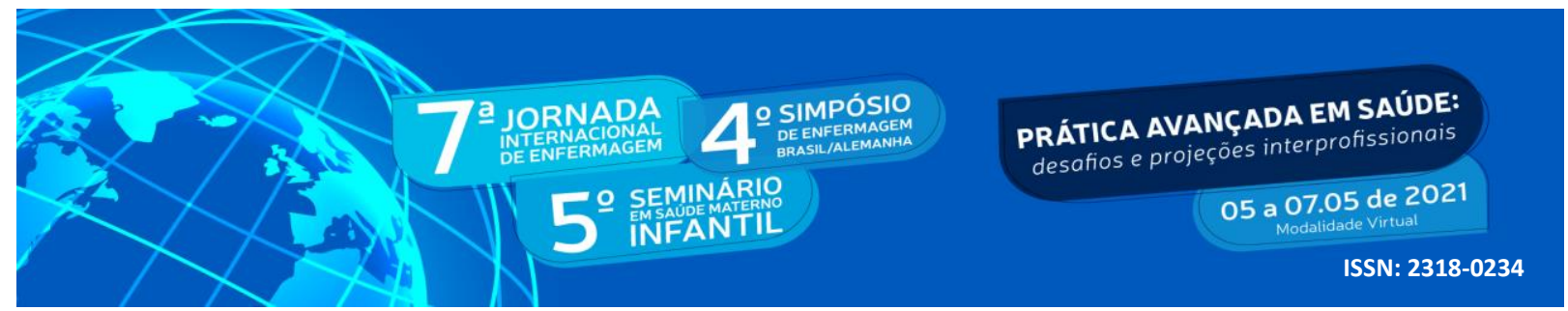

DOI: http://doi.org/10.48195/jie2021-029

\title{
GRUPOS DE GESTANTES ONLINE: ESTRATÉGIA DE INTERVENÇÃO FACE À PANDEMIA DA COVID-191
}

\section{Camila Cioquetta Pereira²; Giovana Luiza Rossato ${ }^{3}$; Leandro da Silva de Medeiros ${ }^{4}$; Victória Friedrich Costa ${ }^{5}$; Denise Comin Silva Almeida ${ }^{6}$; Dirce Stein Backes ${ }^{7}$}

\begin{abstract}
RESUMO
Objetivo: Descrever a dinamização de grupos de gestantes, na modalidade online, como estratégia de intervenção face à pandemia da COVID-19. Método: Trata-se de um estudo descritivo, do tipo relato de experiência, desenvolvido por integrantes do Grupo Gestar, quais sejam, discentes de Enfermagem e residentes do Programa de Residência em Enfermagem Obstétrica da Universidade Franciscana (UFN). Resultados: A implantação do grupo de gestantes, sobretudo, face à pandemia da COVID-19, possibilitou às gestantes e puérperas o compartilhamento de saberes e práticas e o acesso à informação de qualidade. Conclusão: Os grupos de gestantes se constituem em estratégia proativa e prospectiva de educação e promoção da saúde da mulher, bem como o seu empoderamento para o parto e o nascimento.
\end{abstract}

Palavras-chave: Educação em saúde; Gravidez; Infecções por Coronavírus; Saúde materno-infantil; Tecnologia.

\begin{abstract}
Objective: To describe the dynamic of groups of pregnant women, in the online modality, as an intervention strategy in the face of the COVID-19 pandemic. Method: This is a descriptive study, of the type of experience report, developed by members of the Gestar Group, who are nursing students and residents of the Residency Program in Obstetric Nursing of the Franciscan University (UFN). Results: The implementation of the group of pregnant women, mainly, in view of the COVID-19 pandemic, it allowed pregnant women and puerperal women to share knowledge and practices and access to quality information. Conclusion: The groups of pregnant women constitute a proactive and prospective strategy of education and promotion of women's health, as well as their empowerment for childbirth and birth.
\end{abstract}

Key Words: Health education; Pregnancy; Coronavirus infections; Maternal and child health; Technology.

\section{INTRODUÇÃO}

Em 31 de dezembro de 2019, em Wuhan/China, foi detectada a COVID-19 doença associada à infecção de um novo coronavírus denominado SARS-Cov-2 (LANA et al., 2020). A doença com alto poder de transmissão repercutiu em um cenário de pandemia, declarado pela 


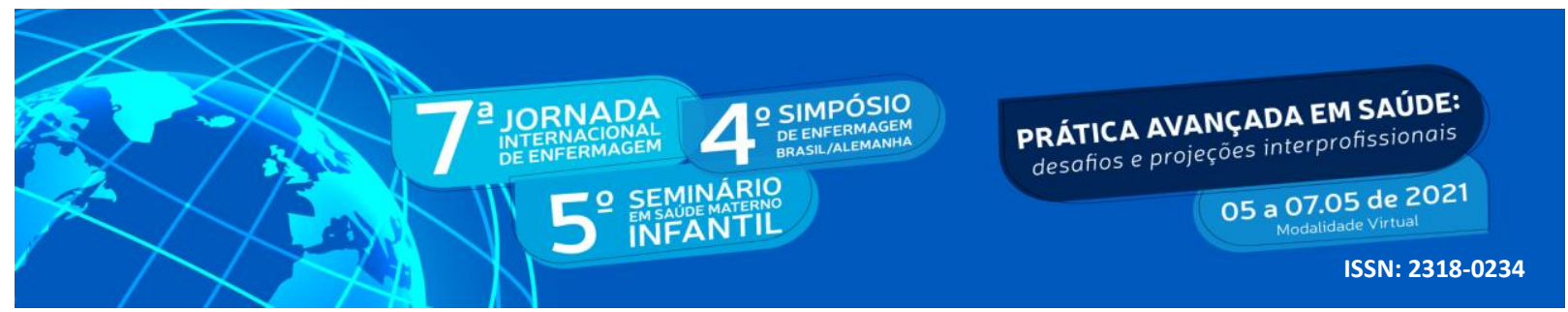

Organização Mundial de Saúde (OMS) como emergência de saúde pública internacional, em 30 de janeiro de 2020 (WHO, 2020).

De acordo com o Ministério da Saúde, o primeiro caso confirmado, no Brasil, foi em 26 de fevereiro de 2020, no estado de São Paulo, apresentando um espectro clínico variando de síndrome gripal e síndrome respiratória aguda grave nos pacientes que foram acometidos pela doença (LANA et al., 2020). Ainda que a COVID-19 se manifeste com sintomas leves na maioria das pessoas, sem muitas complicações, algumas terão a doença de forma grave (CARDOSO et al., 2021).

Sob esse contexto, o Ministério da Saúde (MS) do Brasil incluiu, em abril de 2020, as gestantes e puérperas como grupo de risco à COVID-19, considerando as alterações fisiológicas do ciclo gravídico puerperal (MASCARENHAS et al., 2020). Atualmente, o que tem sido verificado é que as mulheres grávidas com Covid-19 desenvolvem, em sua maioria, quadros leves ou assintomáticos, mas podendo ocorrer também quadros de maior gravidade e fatais (BRASIL, 2020).

A Rede Cegonha, programa estabelecido no âmbito do Sistema Único de Saúde (SUS) proporciona assistência desde o planejamento familiar, com a garantia de um atendimento de qualidade e humanizado para todas as mulheres, com foco na atenção ao parto e nascimento (BRASIL, 2013). Assim, os grupos de gestantes, se constituem em estratégia terapêutica de fácil, rápido e amplo acesso às informações e discussões, sobretudo, às relacionadas à promoção e educação em saúde (ALVES et al., 2019).

Face ao período pandêmico, no entanto, os grupos de gestantes tiveram que ser reordenados e (re)adequados em sua forma de execução, ou seja, de sua modalidade presencial necessitaram de arranjos remotos, na modalidade online. Sob esse enfoque, os questionamentos foram frequentes: Como dinamizar grupos de gestantes na modalidade online, a fim de manter as intervenções face à pandemia da COVID-19. Como despertar o interesse das gestantes e puérperas para a continuidade dos encontros na modalidade online?

\section{OBJETIVO}

Descrever a dinamização de grupos de gestantes, na modalidade online, como estratégia de intervenção face à pandemia da COVID-19. 


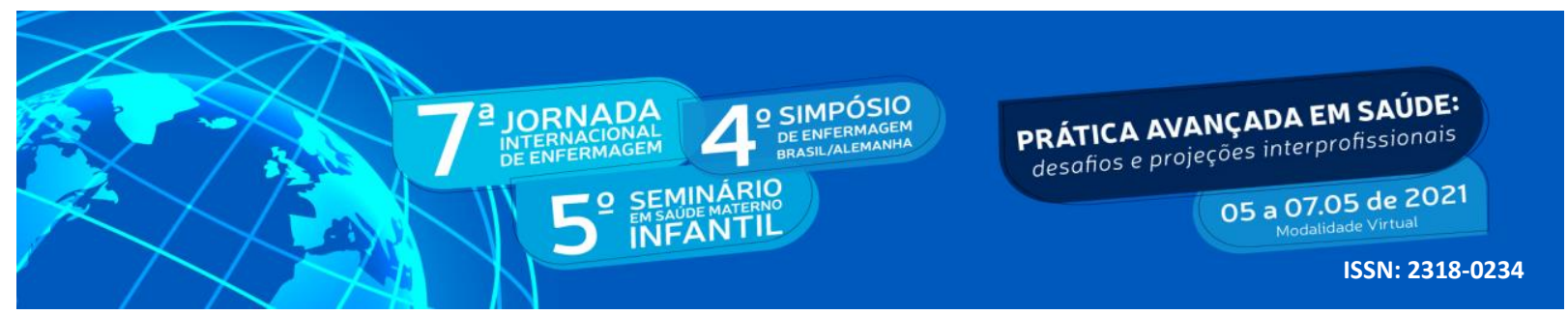

\section{METODOLOGIA}

Trata-se de um estudo descritivo, do tipo relato de experiência, desenvolvido por integrantes do Grupo de Gestantes - GESTAR, quais sejam, discentes de Enfermagem e residentes do Programa de Residência em Enfermagem Obstétrica da Universidade Franciscana (UFN). A decisão pelo desenvolvimento de grupos de gestantes na modalidade virtual surgiu a partir das recomendações e protocolos de prevenção à COVID-19, tais como o distanciamento físico e o isolamento social.

A adoção do isolamento social provocou significativas mudanças nas rotinas locais, inclusive nos serviços de saúde e de educação, nos quais diversas atividades presenciais foram suspensas ou cederam lugar ao formato virtual (SILVA et al., 2020). Diante disso, o Grupo de Gestantes - GESTAR da UFN promoveu seis encontros virtuais com gestantes e puérperas, com pautas previamente discutidas, dos quais serão relatados dois casos, neste estudo.

O grupo GESTAR, foi criado em 2016, com o objetivo de mobilizar uma nova cultura obstétrica entre alunos da graduação da área da saúde, residência em enfermagem obstétrica, alunos do mestrado em Saúde Materno Infantil e usuárias da saúde, em geral, (gestantes e puérperas). Trata-se, portanto, de um Grupo de Estudos e Discussões sobre a Gestação, Parto, Puerpério e outras temáticas de interesse das usuárias de saúde. O grupo se encontrava quinzenalmente, com base em cronograma de atividades semestrais.

\section{RESULTADOS E DISCUSSÃO}

Os grupos de gestantes, sobretudo, em períodos pandêmicos são altamente aconselháveis, considerando que o momento, por si só, gera dúvidas, incertezas e temores. Logo, os encontros se constituem em trocas de experiências e saberes que contribuem para o empoderamento da mulher (gestante e puérpera), além de possibilitarem acesso às informações qualificadas com rapidez e segurança.

O primeiro encontro de gestantes ocorreu no no mês de dezembro de 2020, por meio da rede social Facebook® e transmitido na página da Estratégia Saúde da Família (ESF), Roberto Binato, localizada no município de Santa Maria - RS. A transmissão ocorreu de forma aberta à população e ao vivo, com a seguinte temática: "Direitos da Gestante, Puérpera e Família". 


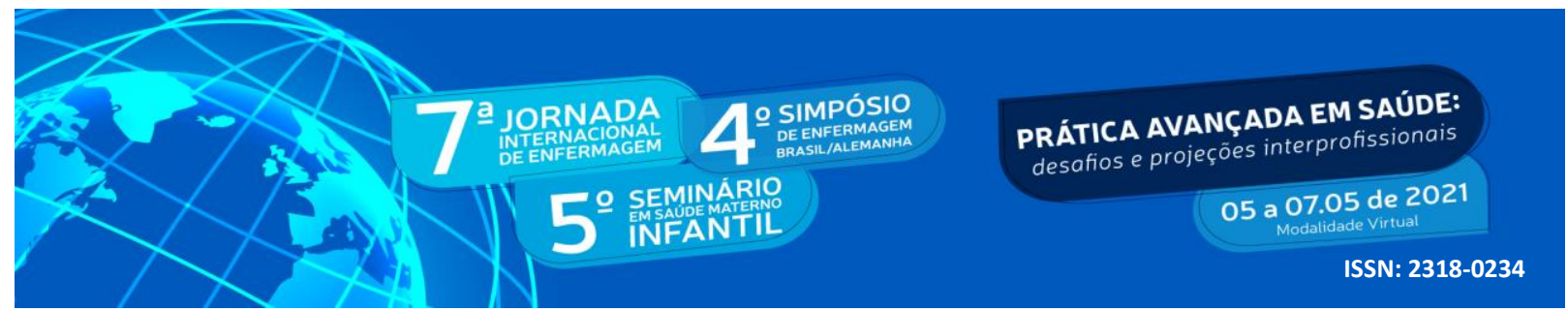

O encontro contou com a participação da enfermeira residente em obstetrícia, uma enfermeira mestre em saúde materno-infantil, uma advogada da área familiarista, uma acadêmica do curso de psicologia e um acadêmico de enfermagem. Em um primeiro momento, a partir da apresentação dos componentes, esclareceu-se a parceria estabelecida entre a Universidade, por meio do GESTAR e a ESF, a qual foi muito bem acolhida entre todos os integrantes.

Iniciou-se, de imediato, a explanação acerca dos direitos das gestantes no que tange a legislação trabalhista. A contribuição da advogada presente no ato foi de suma importância, no sentido de esclarecer, com fundamentação teórica e legal, o direito da gestante à dispensa no trabalho, para as mulheres que têm vínculo empregatício, sobretudo, para as consultas médicas e os exames por no mínimo seis vezes. A profissional esclareceu, ainda, que a gestante poderia participar em todas as consultas recomendadas, sem prejuízos em seu trabalho; a participação do acompanhante às consultas pré-natais; assegurou a licença maternidade de 120 dias, dentre outros assuntos de interesse.

De acordo com o estudo realizado por Ferreira et al (2021), o número de consultas recomendadas e estabelecidas pela Organização Mundial da Saúde (OMS), deve ser igual ou superior a seis. Portanto, é imprescindível o acompanhamento dessa mulher desde o início da gestação, a fim de assegurar uma gravidez saudável não só para a mãe, mas também para o bebê.

Ao discorrer sobre a legislação previdenciária, evidencia-se que o salário maternidade, benefício pago pelo Instituto Nacional do Seguro Social (INSS) à segurada a gestante no período de afastamento de suas atividades, possui durabilidade de 120 dias. Tal serviço pode ser utilizado em casos de a mulher ser empregada ou não, diferido no período de carência, sendo relacionado às contribuições ao INSS (BRASIL, 2020).

Além disso, englobando o direito de família, foi elucidado aos ouvintes sobre as leis que as protegem, não só à mãe, mas também ao recém-nascido, no caso da família não tem o amparo do pai ou do parceiro(a). Ao relatar sobre os direitos que competem à mulher no seu período 


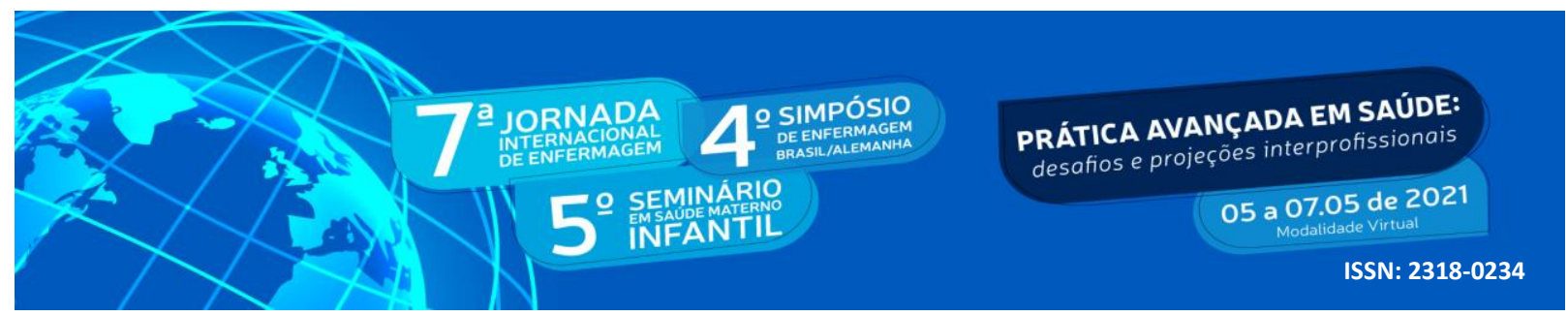

gravídico, nota-se a relevância da explanação para que todas as gestantes tenham em seu alcance essa informação.

Foram, ainda, abordados aspectos sobre o apoio emocional estabelecido pelo acompanhante da mulher no parto e no nascimento, discutido pela acadêmica de psicologia. A Lei do acompanhante $\mathrm{n}^{\mathrm{o}} 11.108$, instaurada desde 2005, determina que os serviços de saúde maternos permitam a presença de um acompanhante de livre escolha da mulher durante todo o período de trabalho de parto, parto e pós-parto imediato (RODRIGUES et al., 2017).

Ficou evidente, na fala da acadêmica, que é essencial o acompanhamento do pai ou parceiro(a) durante as consultas, no processo de parturição, de modo a promover confiança e segurança no momento do parto e do nascimento. No entanto, frente a pandemia há um obstáculo quanto ao acompanhamento do pai ou parceiro (a) durante as consultas e até mesmo durante o parto, pois devido a infecção ativa ou quarentena e as restrições de visitantes em alguns hospitais (HERMANN; FITELSON; BERGINK, 2021).

Nessa perspectiva, a enfermeira convidada abordou algumas questões importantes associadas à primeira consulta de pré-natal da gestante. A mesma destacou que a primeira consulta é a mais importante e prolongada, em razão de que será feita a entrega da Caderneta da Gestante. A Caderneta é um documento fundamental, não só durante o período gravídico, mas em especial para o momento do parto, tendo em vista que o mesmo contém todas as informações da mulher, bem como a sua evolução no momento do parto.

Outro encontro ocorreu em março de 2021, por meio da rede social Facebook®, transmitida pela página da ESF Roberto Binato. Contemplou-se, nesse encontro, a temática "Cuidados com a mulher e o bebê no puerpério". O grupo de gestantes contou com a participação de duas enfermeiras obstétricas e foi mediado por uma residente em enfermagem obstétrica. Em um primeiro momento, a partir da apresentação dos componentes, foi explicado o tema que seria abordado e discutido neste encontro. Salienta-se, que cada um dos encontros teve a participação de cerca de 25 usuárias de saúde (gestantes e puérperas).

Iniciou-se o encontro com a explanação sobre puerpério, termo que se refere ao período que inicia logo após a dequitação da placenta e termina por volta de seis semanas após o parto. 


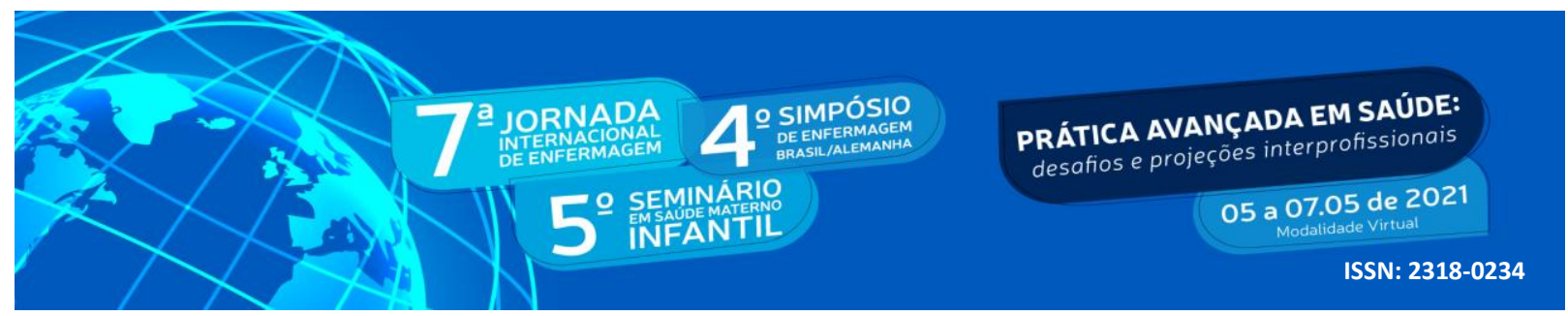

Esse período é marcado por diversas mudanças corporais e adaptações emocionais, que podem resultar em desafios que comprometem a relação mãe-bebê (CASTIGLIONI et al., 2020).

Enfatizou-se, ainda, a importância da atenção à mulher durante o período puerperal, considerando que, não raramente, o cuidado se foca somente no bebê e a mãe acaba ficando em segundo plano. Dessa forma, recomenda-se com ênfase a consulta pós-parto, a fim de identificar fatores influenciáveis na saúde da criança e da mulher e, com isso, seja realizada entre o $7^{\circ}$ a 10 dias do bebê, conforme preconizada pelo Ministério da Saúde (BRASIL, 2013).

A Primeira Semana de Saúde Integral (PSSI), instituída pela Rede Cegonha, considera atividades relacionadas à atenção da saúde das puérperas e dos recém-nascidos. As ações preconizadas neste período e realizadas nos primeiros dias, tem por objetivo a triagem neonatal, a triagem auditiva, a verificação das vacinas BCG e hepatite $B$, além da avaliação do aleitamento materno e, se necessário, orientações e apoio (BRASIL, 2013).

Diante do exposto e a partir desta vivência realizada pelos bolsistas do Grupo GESTAR, na implantação dos grupos de gestantes, defende-se a relevância da manutenção dos encontros de gestantes, mesmo que na modalidade online. A pandemia reiterou, nesse processo, que não há limites de espaços e fronteiras interpessoais, mas vontades coletivas dispostas a cooperarem e fazerem a diferença.

\section{CONCLUSÃO}

A implantação do grupo de gestantes, sobretudo, face à pandemia da COVID-19, possibilitou às gestantes e puérperas o compartilhamento de saberes e práticas e o acesso à informação de qualidade. Os grupos de gestantes se constituem, portanto, em estratégia proativa e prospectiva de educação e promoção da saúde da mulher, bem como o seu empoderamento para o parto e o nascimento.

Considera-se, que para além dos desafios da pandemia, somaram-se oportunidades e possibilidades que, aliadas às demandas da saúde, podem repercutir em boas e novas práticas. Dentre essas, os grupos de gestantes virtuais demonstram-se fortes aliados na promoção e educação em saúde, corroborando com a manutenção do vínculo usuário-serviço.

Além disso, essa vivência contribui para o envolvimento dos bolsistas com a realidade da saúde materno-infantil e o aprimoramento com as tecnologias digitais. Dessa forma, esse 


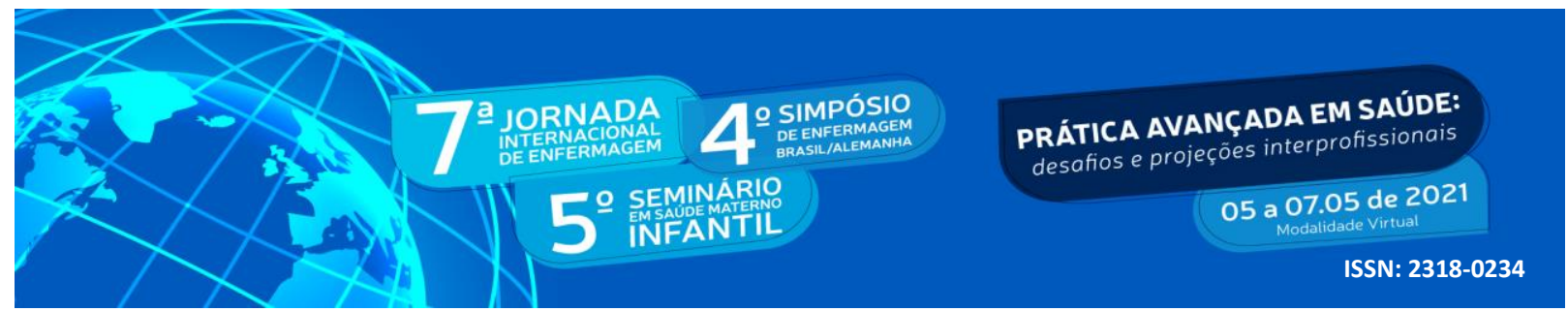

estudo pode vir a sensibilizar outras equipes de saúde para a realização de grupos de gestantes virtuais, no sentido de assegurar à mulher a atenção humanizada durante o ciclo gravídicopuerperal.

\section{REFERÊNCIAS}

ALVES, F. L. C. et al . Grupo de gestantes de alto-risco como estratégia de educação em saúde. Rev. Gaúcha Enferm., Porto Alegre., v. 40, e20180023, 2019.

BRASIL. Ministério da Saúde. Secretaria de Atenção Especializada à Saúde. Departamento de Atenção Hospitalar, Domiciliar e de Urgência. Protocolo de manejo clínico da Covid-19 na Atenção Especializada. 1 ed. rev. Brasília, DF; 2020.

BRASIL. Ministério da Saúde. Manual de Recomendações para a Assistência À Gestante e Puérpera frente à Pandemia de Covid-19. Secretaria de Atenção Primária à Saúde. Departamento de Ações Programáticas e Estratégicas. Brasília, DF. Ministério da Saúde, 2020.

BRASIL. Ministério da Saúde. Gabinete do Ministro. Portaria n ${ }^{\circ}$ 1.459, de 24 junho de 2011. Institui, no âmbito do Sistema Único de Saúde - SUS - a Rede Cegonha. Diário Oficial da União, Poder Executivo, Brasília, DF, 27 jun. 2011.

BRASIL. Ministério da Saúde. Resposta nacional e internacional de enfrentamento ao novo coronavírus. Brasília, DF: MINISTÉRIO DA SAÚDE, 2020. Disponível em: <https://coronavirus.saude.gov.br/index.php/linha-do-tempo >. Acesso em: 01 mar. 2021.

CARDOSO, P. C. et al. A saúde materno-infantil no contexto da pandemia de COVID-19: evidências, recomendações e desafios. Rev. Bras. Saúde Matern. Infant., Recife., v. 21, n. 1, p. 213-220, 2021.

CASTIGLIONI, C. M. et al. Práticas de cuidado no puerpério desenvolvidas por enfermeiras em Estratégias de Saúde da Família. Rev. Enferm. UFSM., v. 10, e50:1-19, 2020.

COELHO, A.L; MORAIS, I. A; ROSA, W. V. S. A utilização de tecnologias da informação em saúde para o enfrentamento da pandemia do Covid-19 no Brasil. Cad. Ibero-amer. Dir. Sanit., Brasília., v. 9, n. 3, 2020.

FERREIRA, G. E. et al. A atenção do enfermeiro na assistência ao pré-natal de baixo risco. Brazilian Journal of Health Review. Curitiba., v.4, n.1, p 2114-2127, 2021.

HERMANN, A; FITELSON, E.M; BERGINK, V. Meeting Maternal Mental Health Needs During the COVID-19 Pandemic. JAMA Psychiatry. v. 78, n. 2, p. 123-124, 2020. 


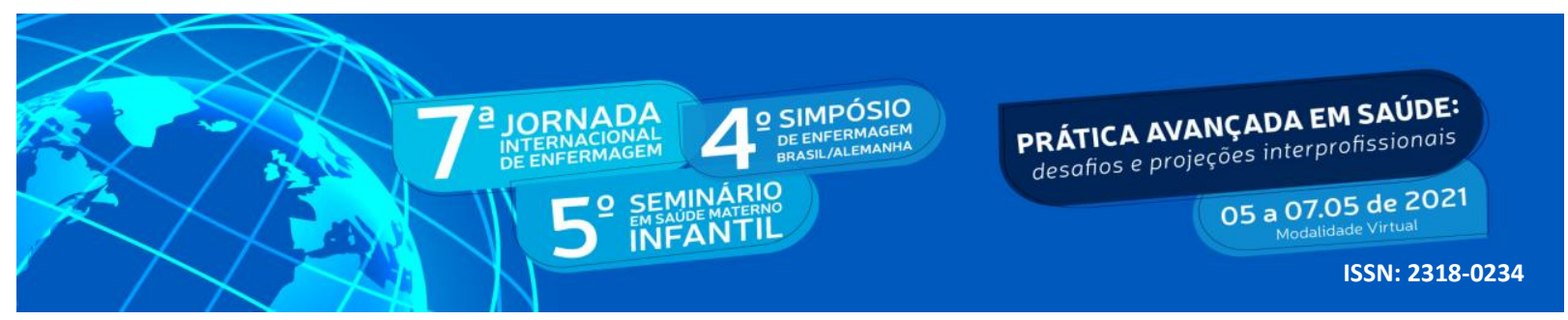

LANA, R. M. et al. Emergência do novo coronavírus (SARS-CoV-2) e o papel de uma vigilância nacional em saúde oportuna e efetiva. Cad. Saúde Pública., Rio de Janeiro., v. 36, n. 3, e00019620, 2020.

MASCARENHAS, V. H. A. et al . COVID-19 e a produção de conhecimento sobre as recomendações na gravidez: revisão de escopo. Rev. Latino-Am. Enfermagem., Ribeirão Preto., v. 28, e3348, 2020.

RODRIGUES, D. P. et al . O Descumprimento Da Lei Do Acompanhante Como Agravo À Saúde Obstétrica. Texto contexto - enferm., Florianópolis., v. 26, n. 3, 2017.

SILVA, M. M. S. et al. Interseção de saberes em mídias sociais para educação em saúde na pandemia de covid-19. SANARE (Sobral, Online)., v. 19, n. 2, p. 84-91, 2020.

WHO. World Health Organization. Coronavirus disease (COVID-2019) situation reports, 2020. Geneva: WHO, 2020. Disponível em: 〈https://www.who.int/emergencies/diseases/novel-coronavirus-2019/situation-reports $>$. Acesso em: 07 de mar. de 2021. 